\title{
SPACE WEATHERING ACROSS THE SOLAR SYSTEM: LESSONS FROM THE MOON AND OUTSTANDING QUESTIONS
}

\author{
Coordinating Author: Michelle S. Thompson \\ Purdue University \\ mthompson@purdue.edu \\ 765-494-8677
}

Co-Authors: Jessica Barnes (University of Arizona), David Blewett (Johns Hopkins Applied Physics Laboratory), Joshua Cahill (Johns Hopkins Applied Physics Laboratory), Brett Denevi (Johns Hopkins Applied Physics Laboratory), Kerri Donaldson Hanna (University of Central Florida), Jeff Gillis-Davis (Washington University St. Louis), Tim Glotch (Stony Brook University), Devanshu Jha (MVJ College of Engineering), Georgiana Kramer (Planetary Science Institute), Nandita Kumari (Stony Brook University), Carey Legett (Los Alamos National Laboratory), Daniel Moriarty (NASA Goddard Space Flight Center), Kurt Retherford (Southwest Research Institute), Shashwat Shukla (University of Twente), Morgan Shusterman (Arizona State University), Indhu Varatherajan (DLR)

Co-Signatories: Caitlin Ahrens (University of Arkansas), Maria E. Banks (NASA Goddard Space Flight Center), Christopher J. Bennett (University of Central Florida), Megha Bhatt (Physical Research Laboratory), Caixia Bu (Columbia University), Kate Burgess (U.S. Naval Research Laboratory), Laura Chaves (Purdue University), Roy Christoffersen (Jacobs/NASA Johnson Space Center), Doug Currie (University of Maryland), Ariel N. Deutsch (Brown University), Luke Dones (Southwest Research Institute), Catherine A. Dukes (University of Virginia), Marina Gemma (American Museum of Natural History), Cesare Grava (Southwest Research Institute), Benjamin Greenhagen (Johns Hopkins Applied Physics Laboratory), Jennifer A. Grier (Planetary Science Institute), Juliane Gross (Rutgers University), Daoru Han (Macau University of Science and Technology), Jose M. Hurtado Jr. (The University of Texas at El Paso), Lindsay Keller (NASA Johnson Space Center), Dara Laczniak (Purdue University), Shuai Li (University of Hawaii), Rafael Martinez (Universidade Federal do Amapá), Francis McCubbin (NASA Johnson Space Center), James McFadden (Purdue University), Heather Meyer (Johns Hopkins Applied Physics Laboratory), Edward L. Patrick (Southwest Research Institute), Carle Pieters (Brown University), Michael J. Poston (Southwest Research Institute), Ujjwal Raut (Southwest Research Institute), Kevin Righter (NASA Johnson Space Center), Marcella Roth (Lockheed Martin), Cassandra Runyon (College of Charleston), Farid Salama (NASA Ames Research Center), Daniel Wolf Savin (Columbia University), Micah J. Schaible (Georgia Institute of Technology), Katherine Shirley (University of Oxford), Timothy J. Stubbs (NASA Goddard Space Flight Center), Jamey R. Szalay (Princeton University), Christian Tai Udovicic (Northern Arizona University), Kathleen Vander Kaaden (Jacobs/NASA Johnson Space Center), Dany Waller (Johns Hopkins Applied Physics Laboratory), Kris Zacny (Honeybee Robotics), Tom Zega (University of Arizona), Xiaoping Zhang (Macau University of Science and Technology) 


\subsection{SPACE WEATHERING AS AN AGENT OF CHANGE ON AIRLESS SURFACES}

Energetic solar wind particles, cosmic rays, and micrometeoroids continually bombard the surfaces of airless bodies [1,2]. Together, these processes alter the microstructure, chemistry, and optical properties of regolith on airless surfaces. These effects are known as space weathering. We only partly understand how regolith is altered from its pristine condition by exposure to space, yet it is the material with which spacecraft and astronauts interact, and the portion of an object most readily observed from orbit or the Earth. Hence, understanding space weathering as a system is critical to scientific understanding and exploration of the solar system (Fig. 1).

The effects of space weathering complicate or even prevent the proper interpretation of compositional remote sensing data collected by orbiting spacecraft at any airless planetary body $[1,2]$. For instance, the connection of meteorites to their parent bodies across the solar system is made more difficult by space weathering (e.g., ordinary chondrites and S-type asteroids). In addition, the role of space weathering in the production (e.g., solar-wind irradiation), destruction (e.g., micrometeorites), and cycling of volatiles on the surface of the Moon and other airless bodies is not well-constrained [e.g., 3-5]. Each of these factors make the study of space weathering a compelling and critical endeavor relevant not only to our understanding of airless surface evolution, but for the interests of future robotic and human exploration across the solar system.

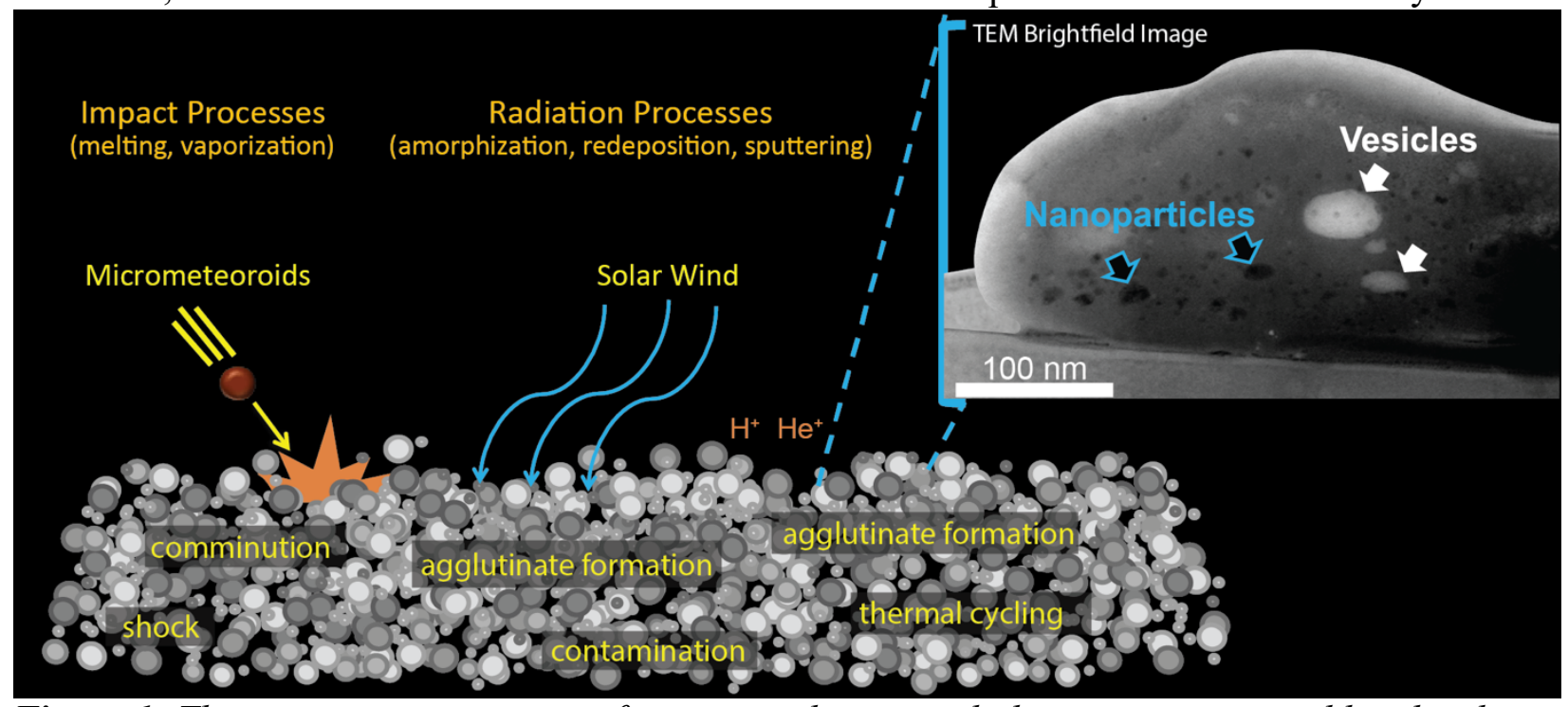

Figure 1: The constituent processes of space weathering including micrometeoroid bombardment and solar wind irradiation as they affect the regolith on airless planetary surfaces. Upper right inset shows the development of microstructural features that result from space weathering including nanoparticles and vesicles.

\subsection{THE LUNAR PERSPECTIVE OF SPACE WEATHERING}

Historically, our understanding of space weathering derives from studies of the Moon. Combining information from returned sample analysis, remote sensing observations, modeling, and laboratory experiments has provided critical insights for our understanding of space weathering as it operates on the lunar surface. Initial identification of the effects of space weathering came via bright, high albedo rays around the freshest craters on the lunar surface. Subsequently, spectral measurements of samples returned by the Apollo missions revealed that soils exposed to the space environment exhibited significantly different spectral properties than soils prepared from compositionally similar, yet unexposed, lunar rocks [1,2]. These observations 
have become the spectral hallmarks of what we now understand to be lunar-style space weathering: darkening (lowered reflectance), reddening (increasing reflectance with increasing wavelength), and the attenuation of characteristic absorption features in spectra across the visible-near infrared (VNIR) wavelength range $(\sim 0.4-3.0 \mu \mathrm{m})$ [2]. In the mid-IR $(\sim 5.0-25 \mu \mathrm{m})$, space weathering causes shifts the silicate Christiansen feature (CF) to longer wavelengths and reduces the strength of the Reststrahlen bands [6,7]. These spectral signatures and their occurrence can be traced to common microstructural and chemical characteristics in returned samples.

The fine particulate nature of regolith and a strong thermal gradient in the upper hundreds of micrometers of regolith complicate mid-infrared measurements. Thermal gradients lead to substantial changes in some mid-IR spectral characteristics, including the CF, Reststrahlen bands, and transparency features relative to spectra acquired from planets with isothermal near-surface environments (i.e., planets with atmospheres). Space weathered surfaces have lower visible albedos that absorb more incident solar light, resulting in a reduced thermal gradient. As such, space weathered airless body surfaces have mid-IR spectra that are markedly different from fresh or less altered surfaces, such as Copernican crater rays and swirls $[6,8,9]$. Similar behavior is observed in laboratory measurements of Apollo 16 feldspathic soils with similar compositions, but with drastically different maturities [6,7].

Laboratory analyses of lunar soils revealed that many of the changes in spectral characteristics are driven by the development of submicroscopic and nanophase Fe particles (npFe) in lunar regolith grains (Fig. 2). Submicroscopic and nanoparticles develop as a result of melt and vaporization processes associated with micrometeoroid impacts and from sputtering and redeposition effects resulting from solar wind irradiation [10]. The particles are hosted both in agglutinate grains, comprised of mineral fragments welded by impact glass, and as surfacecorrelated deposits in grain rims. These particles, produced through space weathering processes, drive significant changes in the spectral characteristics of the material across most wavelengths [e.g., 6,11]. The submicroscopic particles are typically composed of $\mathrm{Fe}^{0}$ (although mixed valence phases do occur), and the spectral effects of these grains vary based on their size, with $<40 \mathrm{~nm}$ grains acting to redden and darken surface spectra and $>40 \mathrm{~nm}$ particles resulting predominantly in spectral darkening [12-14]. However, the size of the npFe in agglutinates in relation to $\mathrm{npFe}$ found in amorphous rims has a large uncertainty. The $\mathrm{npFe}$ found in agglutinates have been reported to be (1) similar in size to those found in amorphous rims [10]; (2) at least twice as large as those found in amorphous rims, (i.e., averaging $\sim 7 \mathrm{~nm}$ vs. $\sim 3 \mathrm{~nm}$ for the rims) [11]; or agglutinitic npFe may be (3) significantly larger, averaging over $100 \mathrm{~nm}$ in diameter [15].

The microstructural signatures of space weathering in lunar soils are not limited to $\mathrm{npFe}$, however, and also include vesiculated textures, partially and completely amorphous grain rims produced via solar wind irradiation, tracks of disrupted crystal structure in soil grains caused by bombardment with high energy solar flare particles, and melt and vapor redeposition on grain exteriors formed through micrometeoroid bombardment [10]. While the origins of these features were attributed to individual constituent space weathering processes, our understanding of how these alteration mechanisms work was improved substantially by the development of laboratory techniques that simulate airless body surface processes.

To investigate the development and evolution of the optical, chemical, and microstructural characteristics of space weathering, experiments were designed to simulate these processes in the laboratory. To simulate the short duration, high-temperature thermal events characteristic of micrometeoroid bombardment, pulsed-laser irradiation was employed [e.g.,16-18]. Using silicate 
minerals commonly found in lunar samples as targets, these experiments resulted in the development of spectral and microchemical signatures similar to naturally-space weathered lunar samples (e.g., reddened and darkened spectral slopes and the production of $n p F e$ grains). To simulate the effects of solar wind irradiation, ion bombardment has been performed, frequently using ions and energies that are appropriate for the solar wind [e.g., 19-21]. Ion irradiation experiments, also performed predominantly on silicate minerals, have resulted in

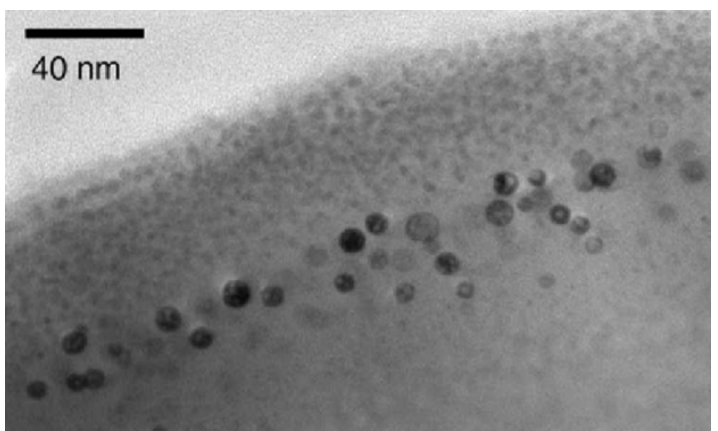
Figure 2: Transmission electron microscope image of npFe (dark changes in spectral properties of the samples and spherules) in the rim of a lunar soil grain.
provided context for the sputtering and redeposition of material on grain surfaces, the production of amorphous rims, and the development of vesiculated textures in natural samples. Together, these novel laboratory experiments have provided significant advances towards understanding the development of space weathering characteristics on the Moon, the role of each constituent weathering processes, and their associated effects. However, despite decades of spectral observations, modeling, returned sample analyses, and laboratory experiments, there remain significant outstanding questions about the nature of space weathering on the lunar surface and across the solar system.

\subsection{PERSISTENT MYSTERIES OF THE LUNAR SURFACE}

Our understanding of the Moon and its active processes has evolved significantly over the last several decades. Recent discoveries have both posed new questions and reignited interest in knowledge gaps which are relevant to and critical for continued exploration of the Moon via orbital, robotic, and crewed missions. Here, we highlight those of top importance.

\subsection{POSSIBLE SPACE WEATHERING CONTRIBUTIONS TO THE VOLATILE} INVENTORY OF THE MOON

The "bone-dry" paradigm of the Moon no longer holds following the discovery of 'water' on both the lunar surface and in returned lunar samples. The examination of spectral reflectance data from multiple remote sensing spacecraft, including the Moon Mineralogy Mapper $\left(\mathrm{M}^{3}\right)$ aboard the Chandrayaan-1 orbiter, detected a $\sim 3 \mu \mathrm{m}$ absorption band on the lunar surface, likely due to the presence of water (as $\mathrm{OH}$ and/or $\mathrm{H}_{2} \mathrm{O}$ ) [e.g., 22-25]. In addition, recent laboratory measurements of returned samples and lunar meteorites have confirmed the presence of water (both $\mathrm{OH}$ and $\mathrm{H}_{2} \mathrm{O}$ ) in lunar volatile-bearing phases [26], volcanic glasses [27], melt inclusions [27], in the interiors of agglutinates [28], and in nominally anhydrous minerals [29]. One possible source for lunar surface water has been identified as the interaction of solar wind protons $\left(\mathrm{H}^{+}\right)$with O-bearing surface minerals to form water. There is strong evidence supporting this hypothesis from $\mathrm{M}^{3} \mathrm{OH} / \mathrm{H}_{2} \mathrm{O}$ maps of the lunar swirls (see Section "Revealing the Relative Contributions of Solar Wind and Micrometeoroids to Space Weathering"). Experiments and laboratory measurements have demonstrated this process is capable of forming water, but our understanding of its efficacy or contribution to the volatile inventory of the Moon is at an early stage [e.g., 30].

As a community, we have yet to definitively determine the spatial distribution and abundance of water and other volatiles at the lunar surface and whether or not it is correlated with, e.g., latitude, surface composition, location, and/or time of day. Developing an understanding of the source(s) of water found on the surface of the Moon and their relationship to space weathering 
processes is of critical importance for continued exploration efforts. Recent studies have indicated that volatile cycling on the Moon, including the transportation to and deposition of terrestrial oxygen at the lunar poles, has resulted in the production of unique and unexpected mineralogies, including hematite $\left(\mathrm{Fe}_{2} \mathrm{O}_{3}\right)$ [31]. Converging on a model of the lunar surface volatile cycle and understanding the role of solar wind as a source of water generation and resupply is crucial for future in-situ resource utilization (ISRU) and human exploration initiatives.

Among the implanted solar wind volatiles on the Moon, Helium-3 $\left({ }^{3} \mathrm{He}\right)$ is another valuable resource because of its potential, as a fusion fuel, to drive the Earthly and cislunar energy market. The retention of solar wind implanted ${ }^{3} \mathrm{He}$ is strongly dependent on the abundance of electroconductive minerals in the regolith and its degree of maturation [32]. Under the influence of surface solar radiation, electroconductive metallic minerals (e.g., ilmenite) do not become amorphous compared to other rock-forming minerals, thereby suggesting high thermal and mechanical stability of implanted ${ }^{3} \mathrm{He}$. As solar wind is a major contributor to regolith evolution, the ${ }^{3} \mathrm{He}$ concentration of retained solar wind gases is significant in the surface layers of individual grains. Over time, comminution increases the surface area to volume ratio of regolith grains providing increased opportunities for interactions between solar wind and fresh grain surfaces, resulting in greater abundance of retained gas species.

Direct laboratory measurements of lunar samples demonstrate a strong correlation between ${ }^{3} \mathrm{He}$ content (in ppb), maturity index, and $\mathrm{TiO}_{2}$ concentration (in wt $\%$ ) that is extrapolated to understand the spatial distribution of ${ }^{3} \mathrm{He}$ at both regional and global scales using remote sensing data [33-35]. More recently, the spectral effects of space weathering were employed to reveal minute variations in maturity trends associated with implanted ${ }^{3} \mathrm{He}$ from high resolution $\mathrm{M}^{3}$ data. It was found that reduced spectral contrast due to attenuation of VNIR absorption features is highly correlated with the retention of implanted ${ }^{3} \mathrm{He}$ compared to other weathering effects. Moreover, a measure of variability between extracted ${ }^{3} \mathrm{He}$-rich samples in spatial domain represents cyclic pattern in the variogram trends, indicative of the occurrence of episodic space weathering events over geological timescales. From prior studies, pyroclastic regolith showcases great exploration potential of harboring near-surface reservoirs of both ${ }^{3} \mathrm{He}$ and water. However, one of the limitations in such modeling initiatives is ground truth data; more samples from nearside and, in particular, the farside are required to effectively address spatial variability and saturation effect, thereby leading to the re-calibration of models. It is also important to identify and characterize the stochastic nature of weathering processes (in space and time) governing the emplacement and evolution of high-grade high-tonnage volatiles at the mining scale that could be of interest for both science and necessary for human (or robotic) exploration.

\subsection{REVEALING THE RELATIVE CONTRIBUTIONS OF SOLAR WIND AND} MICROMETEOROIDS TO SPACE WEATHERING

A long-standing question is the relative role of micrometeoroids versus solar wind in the space weathering of planetary regoliths. In returned samples we have observed microstructural and chemical features attributed to each of these constituent processes (e.g., amorphous grain rims resulting from solar wind irradiation and melt deposits and microcraters formed through micrometeoroid impacts). However, there has been significant debate regarding their relative roles in contributing to the optical signatures of space weathering. Understanding which process is dominant and is driving changes in microstructure and spectral properties is important for an improved understanding of regolith development on the lunar surface, and for expanding our model of space weathering beyond the Moon. Dust and solar wind flux vary as a function of heliocentric distance and knowing their relative contributions to the weathering of airless surfaces 
may enable the prediction and attribution of optical changes to particular processes on planetary surfaces from which we do not yet have returned samples.

Lunar swirls are sinuous, high albedo features, that mimic some properties of optically immature soils. Their curvilinear shape is often accentuated by low albedo regions (dark lanes) in the visible that weave between the bright swirls. Swirls on the maria are characterized by strong albedo contrasts and complex, sinuous morphology, whereas those on highland terrain may be less prominent and exhibit simpler shapes such as single loops or diffuse bright spots. Coincident with all lunar swirls are regions of strong crustal magnetic fields $(\geq \sim 2$ nT) $[36,37]$ and the high albedo swirl features are suggestive of low optical maturity. Swirls have been identified on the lunar maria
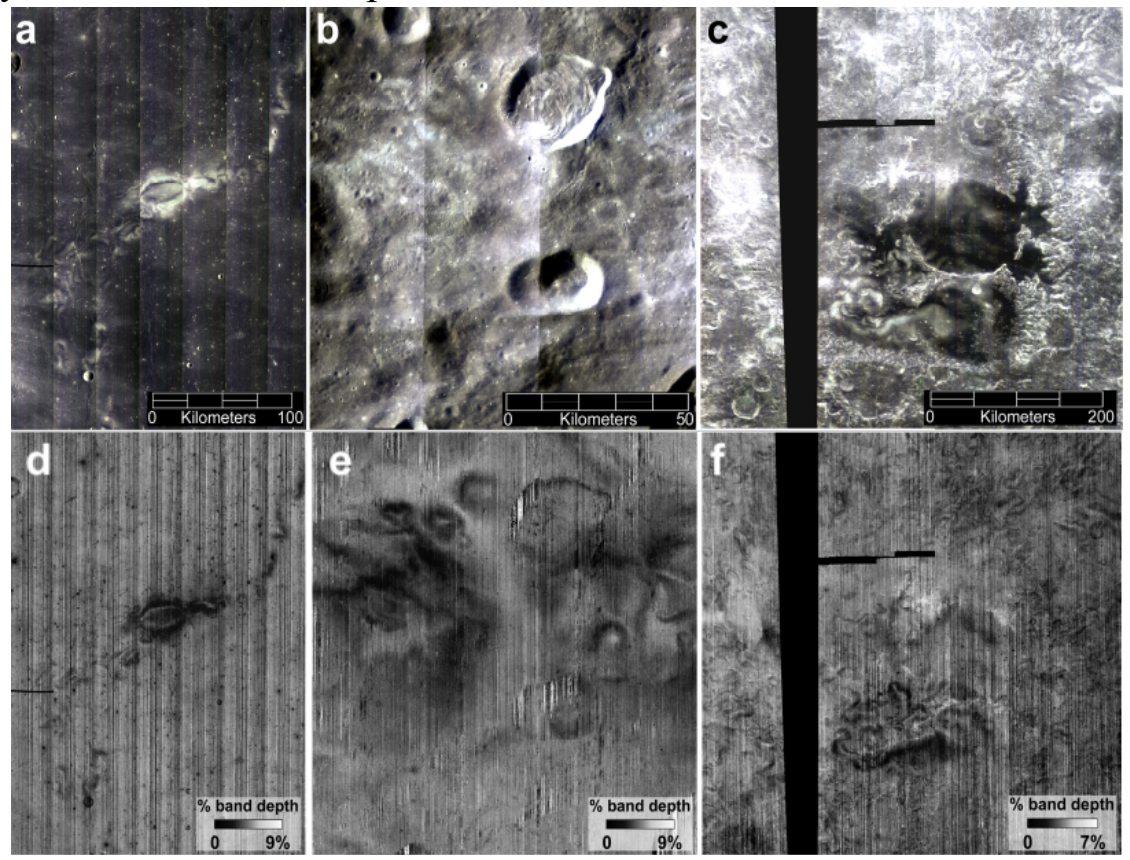

Figure 3: (Top) $M^{3}$ mosaics in true color $(R=700 \mathrm{~nm}, G=580$ $\mathrm{nm}, \mathrm{B}=460 \mathrm{~nm}$ ) of three swirl regions: (a) Reiner Gamma, (b) Gerasimovich, and (c) Mare Ingenii. (Bottom) Parameter images of the depth of the $2.82 \mu \mathrm{m}$ absorption feature. This parameter shows the relative $\mathrm{OH} / \mathrm{H}_{2} \mathrm{O}$ abundance of the swirl region above each: (d) Reiner Gamma, (e) Gerasimovich, and (f) Mare Ingenii. The swirls are recognizable by their shape and low OH abundance. and highlands and are not directly related to a specific lithology [38-40]. Lunar swirls also impart no topographic expression or anomalous surface roughness [8,41]. All of these characteristics and decades of analysis using a wide variety of measurement techniques support the hypothesis [36] that lunar swirls are locations where the magnetic fields shield the surface from some solar wind irradiation which in turn affects soil evolution.

Models indicate that the Moon's crustal magnetic anomalies are strong enough to interact with and deflect charged, low-mass solar wind particles (protons and electrons) away from the swirls $[42,43]$, which may affect their spectral characteristics, and explains the observation that high albedo swirls are depleted in $\mathrm{OH} / \mathrm{H}_{2} \mathrm{O}$ compared with surrounding regolith. Moreover, some of the deflected particles are predicted to be funneled onto the dark lanes [37,44], causing them to experience distinctive weathering processes [45]. However, the magnetic fields have little to no effect on incident micrometeoroids, so swirl surfaces do weather, just at a significantly reduced rate $[8,45,46]$. Some remote sensing data analyses of lunar swirl regions suggest that solar wind irradiation plays the dominant role in driving space weathering on the lunar surface $[45,47]$. However, analysis of returned samples and laboratory analog experiments indicate that micrometeoroid bombardment does play a significant role in space weathering at the Moon [11]. Here lies the conundrum. Our understanding of airless surfaces across the solar system depends on the development of a comprehensive model for space weathering. As such, improving our understanding of the contributions of constituent processes is critical for broadening our 
perspective of space weathering beyond the Moon, and lunar swirls provide a unique location to examine the relative roles of solar wind and micrometeoroid bombardment on surface maturation.

\subsection{EXPANDING OUR UNDERSTANDING OF SPACE WEATHERING BEYOND} THE MOON: A CRITICAL TOOL FOR EXPLORATION

Space weathering affects the surfaces of all airless bodies in the solar system. While our understanding of this phenomenon is built on our observations of the Moon, it is critical that we expand our model for space weathering beyond the lunar surface. Our ability to accurately interpret compositions of planetary surfaces from remote sensing data relies on our understanding of space weathering. For example, the proposed link between S-type asteroids and ordinary chondrites was confirmed this past decade through studies of the samples returned by the Hayabusa mission [48]. Analysis of dust grains from asteroid Itokawa revealed novel characteristics of space weathering including FeS nanoparticles (npFeS). Such observations expanded our understanding of space weathering processes, indicating that the microstructural and chemical products of solar wind and micrometeoroid bombardment are dependent on the target composition. Similarly, recent experiments and observations have indicated that the thermal environment may play a role in the development and progression of space weathering characteristics [49]. Finally, our model has grown even more complex with the completion of experiments simulating space weathering of carbonaceous chondrites in advance of sample return from asteroids Bennu and Ryugu, the targets of the OSIRIS-REx and Hayabusa2 missions, respectively. Using ion irradiation and pulsed-laser irradiation to simulate weathering in the laboratory, results have shown both similarities and differences between space weathering of these materials and lunar samples [e.g.,50-52]. While nanoparticles do develop as a result of simulated space weathering of carbonaceous materials, there is more compositional diversity than is observed in lunar or even ordinary chondritic samples, including $\mathrm{Fe}^{0}, \mathrm{FeS}, \mathrm{FeNiS}$, and $\mathrm{Fe}_{3} \mathrm{O}_{4}$. The variety of nanoparticle phases, likely a result of the compositional diversity of the target, cause unpredictable spectral effects. Experimental samples have shown both spectral reddening and bluing, spectral darkening and brightening as a result of simulated weathering. These results indicate that the well-understood spectral and microstructural effects of lunar-style space weathering do not apply to all planetary regoliths.

\subsection{RECOMMENDATIONS}

Maximizing the science return from ongoing and future missions relies on an improved understanding of how compositional and mineralogical diversity affects space weathering signatures. The role of volatile phases, ices, and organics and the effect of temperature in space weathering across the solar system is still poorly understood. Studies of space weathering are and will be critical for the interpretation of remote sensing data from any mission to an airless planetary surface, including past and current targets such as Itokawa, Bennu, Ryugu, Vesta, Ceres, and Mercury. Similarly, this work will be of paramount importance in the exploration of new worlds with robotic spacecraft, including Phobos, Deimos, and moons of the outer solar system. We must continue to improve our understanding of space weathering through coordinated, multifaceted techniques including remote sensing, modelling, laboratory experiments, and the analysis of returned samples, including surface soils. Though significant progress has been made towards developing a model for space weathering across the solar system, outstanding questions remain. To maximize the science return of past, ongoing, and future missions and to pave the way for continued orbital, robotic, and human exploration of the solar system we recommend: 
1. Prioritize understanding the space weathering environment at the Moon as a natural laboratory for other airless bodies (See companion white paper 'Key questions in regolith development on planetary bodies: depth, distribution, processes').

2. Consider missions focused on lunar swirls to better constrain the contributions of various constituent processes to space weathering (See companion white papers 'Science Case for a Lander or Rover Mission to a Lunar Magnetic Anomaly and Swirl', 'Next Generation Lunar Orbiter', and 'The Plethora of Science Afforded by a Lunar Swirl').

3. Invest in studies linking space weathering to the production and cycling of volatile deposits relevant to human exploration and in situ resource utilization.

4. Prioritize landed and orbital missions which better characterize the space weathering environment as relevant to exploration hazards (See companion white paper 'Assessing the Present-Day Impact Flux to the Lunar Surface via Impact Monitoring and its Implications for Sustained Lunar Exploration').

6.0 REFERENCES

[1] Hapke B. 2001. J. Geophys. Res. Planets 106:10039-10073. [2] Pieters C. M. and Noble S. K. 2016. J. Geophys. Res. Planets 121:1865-1884. [3] Greenwood J. P. et al 2011. Nature Geosci. 4:79-82 [4] Liu Y. et al. 2012. Nature Geosci. 5:779-782. [5] Bandfield, J. L. et al. 2018. Nature Geosci. 11:173-177. [6] Lucey P. G. et al. 2017. Icarus 283:343-351. [7] Donaldson Hanna K. L. et al. 2017. Icarus 283:326-342. [8] Glotch T. D. et al. 2015. Nature Comm. 6:1-8. [9] Kumari N. et al. 2020. LPSC abstract \#1967. [10] Keller L. P. and McKay D. S. 1997. Geochim. Cosmochim. Acta 61:2331-2341. [11] Denevi B. W. et al. 2014. J. Geophys. Res. Planets 119:976-997. [12] Keller L.P. and Clemett, S. J. 2001. LPSC Abstract \#2097. [13] Noble S. K. et al. 2007. Icarus 192:629-642. [14] Thompson M.S. et al. 2016. Meteorit. Planet. Sci. 51:1082-1095. [15] Basu A., 2005. J. Earth Sys. Sci. 114:375-380. [16] Sasaki S. et al. 2001. Nature 410:555-557. [17] Brunetto R. et al. 2006. Icarus 180:546-554. [18] Loeffler M. et al. 2016. Meteorit. Planet. Sci. 51:261-275. [19] Loeffler M. et al. 2009. J. Geophys. Res. Planets 114:E3. [20] Lantz C. et al. 2017. Icarus 285:43-57. [21] Dukes C. et al. 1999. J. Geophys. Res. Planets 104:1865-1872. [22] Pieters C. M. et al. 2009. Science 326:568-572. [23] Sunshine J. M. et al. 2009. Science, 326:565-568. [24] Klima R. et al. 2013. Nature Geosci. 6:737-741. [25] McCord T. B. et al. 2011. J. Geophys. Res. Planets 116:E6. [26] McCubbin F. M. et al. 2010. Proc. Nat. Acad. Sci. 107:11223-11228. [27] Saal A. E. et al. 2013. Science 340:1317-1320. [28] Liu Y. et al. 2012. Nature Geosci. 5:779-782. [29] Hui H. et al. 2013. Nature Geosci. 6:177-180. [30] Bradley J. P. et al. 2014. Proc. Nat. Acad. Sci. 111:1732-1735. [31] Li S. et al. 2019. LPSC Abstract \#2132. [32] Swindle T. D. et al. 1990. UA/NASA Space Eng. [33] Johnson J. R. et al. 1999. Geophys. Res. Lett. 26:385-388. [34] Fa W. Z., and Jin Y. Q. 2007. Icarus 190:15-23. [35] Slyuta E. N. et al. 2007. LPSC Abstract \#2175. [36] Hood L. L. and Schubert G. 1980. Science 208:49-51. [37] Poppe A. R. et al. 2016. Icarus 266:261-266. [38] Pinet P. C. et al. 2000. J. Geophys. Res. 105:9457-9476. [39] Blewett D. T. et al. 2002. J. Geophys. Res. Planet. 116:E2. [40] Denevi B. W. et al. 2016. Icarus 273:53-67. [41] Neish C. D. et al. 2011. Icarus 215:186-196. [42] Harnett E. M. and Winglee R. M. 2002. J. Geophys. Res. Space Phys. 107:1421. [43] Deca J., D. et al. 2020 J. Geophys. Res. Planet. 125:e2019JE006219. [44] Bamford R. A. et al. 2016. Astrophys. J. 830:146. [45] Kramer G. Y. et al. 2011a. J. Geophys. Res. Planets 116:E04008. [46] Kramer G. Y. et al. 2011b. J. Geophys. Res. Planets 116:E00G18. [47] Denevi B. W. et al. 2014. J. Geophys. Res. Planet. 119:976-997. [48] Nakamura T. et al. 2011. Science 333:1113-1116. [49] Lucey P.G. et al. 2014. J. Geophys. Res. Planet. 119:1665-1679. [49] Lantz C. et al. 2017. Icarus 285:43-57. [50] Gillis-Davis J. J. et al. 2017. Icarus 286:1-14. [51] Thompson M. S. et al. 2019. Icarus 319:499-511. 